\title{
Nobel Prize in Physics 1972
}

\section{Peter, University of Geneva}

The 1972 Nobel Prize in Physics was awarded to J. Bardeen, L.N. Cooper and J.R. Schrieffer for their theory of superconductivity. The article by the laureates of 1957 (Phys. Rev. 108, 1175) rendered the hitherto elusive phenomenon of superconductivity accessible to theoretical understanding. In five sections, the authors' microscopic theory describes the superconducting ground state, the energy gap, the excited states and the thermodynamic properties. The magnitude of the break-through must be measured against the prior state of the art.

The original discovery by Kamerlingh Onnes (1911) had been followed by much experimental work, leading to the discovery of the Meissner effect (1933), of Type II superconductivity (1937) and of the isotope effect (1950).

The Gorter-Casimir model (1934), the London theory (1935), the LandauGinzburg equations (1950) and the Pippard equations (1953) were known theoretically. Microscopic theories were attempted based on electronelectron interactions, notably by Welker (1939) for magnetic interactions, by Heisenberg and Koppe (1950) for Coulomb interactions and by Fröhlich (1950) for electron-phonon interactions. Schafroth (1951) pointed out that the Meissner effect could not be obtained by perturbation theory starting from independent electrons, and he formulated with Butler and Blatt a theory (1954) based on pairs separated in space. In 1956, Volume $X V$ of the Encyclopedia of Physics appeared with an article by Bardeen on superconductivity where he states:

"In spite of the large amount of excellent experimental and theoretical work devoted to the problem, there remain major unsettled questions. However, the area in which the answers must be found has been narrowed considerably".

In 1956 also, Cooper, one of Bardeen's students, treated a soluble problem of two interacting electrons above a non-interacting Fermi sea, leading to a lowering of the energy of the pair which was non-analytic in the pair interaction. Schrieffer, also a student of Bardeen's, adapted the

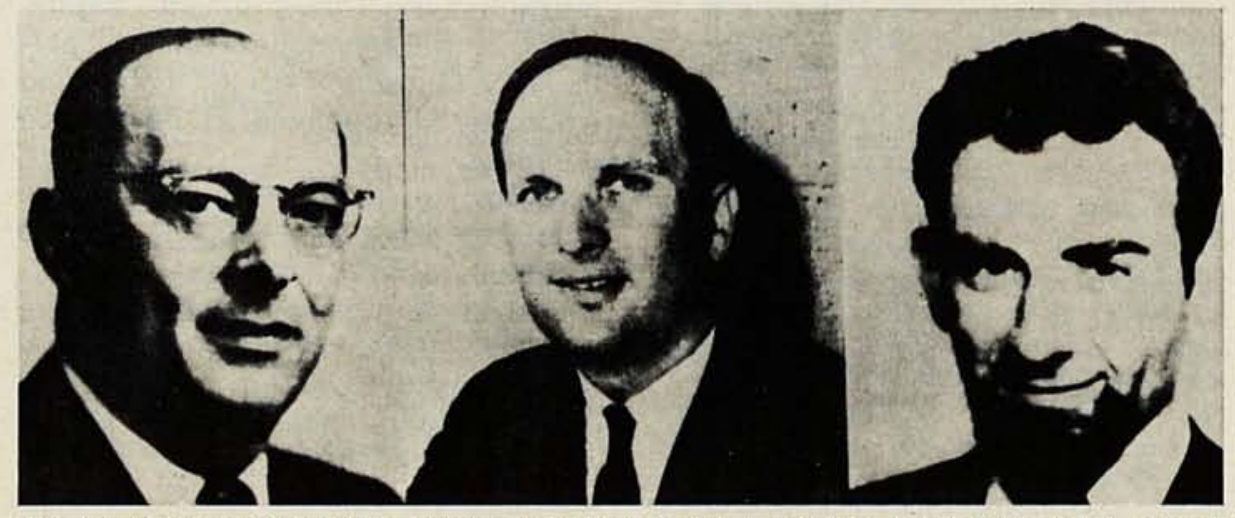

The 1972 Nobel Prize winners in Physics (from left to right): J. Bardeen, J. Schrieffer, and L. Cooper.

intermediate coupling approximation of Tomonaga (1947) to the treatment of Cooper pairs. After this, the complete BCS theory was written within an astonishingly short time.

This short review of : the events leading to the BCS paper allows a guess at the essential virtues of the BCS team: they were familiar with the known-how of their time, decided what was essential to their problem, recognized how it could be solved, carried the solution through to the end and carefully compared their results with the existing experimental work.

What was left to be done after this breakthrough? The interaction between pairs was chosen by BCS both ingeniously and somewhat arbitrarily in order to facilitate the mathematical treatment while retaining the essential features of a phonon-induced interaction, particularly the isotope effect. Field theoretical methods introduced by Gorkov (1958) to the problem permitted Nambu and Eliashberg (1960) to use more realistic retarded interactions, including the Coulomb repulsion. Josephson (1961) predicted supercurrents in junctions with their intriguing quantum interference effects.

Experimental physicists were greatly stimulated by the work of BCS. The energy gap was demonstrated by tunneling (Giaever, 1960), the superconducting flux quantum was measured in 1961 by Daever and Fairbank and Doll and Näbauer, the Josephson effect in 1962 by Anderson and Rowell.
Slowly, superconductors are also finding practical application. Following the work of Abrikosov (1957), Type II superconductors have been studied with regard to their high critical fields. The superconductor with the highest transition temperature is presently $\mathrm{N}_{0.75} \mathrm{Al}_{0.20} \mathrm{Ge}_{0.05} ; \mathrm{T}_{\mathrm{c}}=21.05 \mathrm{~K}$ (Matthias 1967 and Ruzicka 1970).

The Josephson effect as well as recent work on the non-equilibrium properties of superconductors might lead one day to applications concerning acquisition and storage of information. Superconductivity offers ways of handling both the highest and the lowest magnetic fields.

Whatever the future development of superconductivity will be, the central part played by BCS is abundantly clear. In view of this evidence, the Nobel Prize Committee had to innovate and to award to one of this year's laureates, J. Bardeen, his second prize in physics. It has surely made a fortunate choice.

\section{High Energy and Particle Physics Division}

The officers of the new Board were elected on the occasion of the Second General EPS Conference in Wiesbaden :

Chairman : M. Froissart (Gif/Yvette)

Secretary: G. Charpak (Geneva)

Members: G. Bernardini (Pisa)

J. Hamilton (Copenhagen)

H. Lipkin (Rehovoth)

W. Paul (Bonn) 